\title{
Low-Voltage Plasma Generator Based on Standing Wave Voltage Magnification
}

\author{
Mihnea Antoniu Covaci and Lorant Andras Szolga * (1D
}

Optoelectronics Group, Basis of Electronics Department, Faculty of Electronics, Telecommunications, and Information Technology, UTCN (Technical University of Cluj-Napoca), 400114 Cluj-Napoca, Romania; covaci.a.mihnea@utcluj.didatec.ro

* Correspondence: lorant.szolga@bel.utcluj.ro; Tel.: +40-744-490923

\begin{abstract}
The applied electronics domain has great importance due to many applications, such as energy conversion, directly influencing specific processes involving renewable energy. The development of newer manufacturing processes for many integrated components allows for better overall efficiency in certain switching DC/DC converters used for implementing such low-voltage electric field or X-ray generators. Hence, the work presented in this paper involves the development of a helical resonator using a complex DC/DC low-voltage power supply and other required high-voltage conversion circuits. It also follows that there is a possibility of improving this design using only renewable energy supplies. Following two different approach methods, using a circuit model compared to transmission line mathematics, the standing wave propagation mathematics yields multiple scenarios for building a model that predicts the secondary side natural frequency. Moreover, standing wave occurrence conditions in various-dimensioned conductors were further investigated.
\end{abstract}

Keywords: helical resonator; standing wave; power electronics; renewable energy

Citation: Covaci, M.A.; Szolga, L.A. Low-Voltage Plasma Generator Based on Standing Wave Voltage

Magnification. Sustainability 2022, 14, 2890. https://doi.org/10.3390/ su14052890

Academic Editor: Nikos

E. Mastorakis

Received: 12 January 2022

Accepted: 25 February 2022

Published: 2 March 2022

Publisher's Note: MDPI stays neutral with regard to jurisdictional claims in published maps and institutional affiliations.

Copyright: (c) 2022 by the authors. Licensee MDPI, Basel, Switzerland. This article is an open access article distributed under the terms and conditions of the Creative Commons Attribution (CC BY) license (https:// creativecommons.org/licenses/by/ $4.0 /)$.

\section{Introduction}

Helical antennas, also known as helical resonators, are gaining increased interest nowadays. Newer semiconductor manufacturing processes allow using such designs in closed-loop modes by measuring the number of generated X-rays using CdS/CdTe sensors [1]. Such configurations could have various medical or military applications, where $\mathrm{X}$-ray or ozone generators are required.

The development of the compact electric field and X-ray generators has become a significant challenge regarding the overall system efficiency and spatial constraints. The implemented system focuses on a hardware solution; the helical resonator acts as a quarterwave oscillator which makes possible the achievement of high voltages by operating the resonator close to its resonant frequency. It also obtains a standing wave inside the conductor, composed of two components-the incident wave and the reflected wave.

Beyond alternative methods, such as using an approximate equation form of one of Maxwell's laws, similar designs were used in satellite communications [2,3]. Such approximation leads to a high tolerance in mathematical calculations; the approach presented in this study considers obtaining standing waves inside the helical resonator winding wire. In contrast, using Maxwell's equations, the propagation medium with standing waveforms is the waveguide formed by the helical-coil winding air core, with the plasma at the top load providing the required reflection factor. Moreover, having such a low operating voltage makes it possible to power such resonators using only renewable energy, such as solar panels or geothermal probes.

The main phenomenon used in finding the natural frequency through mathematics is the standing wave. It operates similarly to constraints that appear in SMD circuit designs at very high frequencies-experimentally, reflections are more pronounced along the transmission line as the frequency or line length increases. 
The helical resonator presented here is formerly known as the Tesla coil. By operating the secondary coil close to its natural frequency, the signal propagating towards the top load becomes trapped due to reflection coefficients. During this operation regime, a transition from the inductor to resonator occurs by overlaying the incident wave with the reflected wave, also known as standing-wave summation. Thus, due to constructive and destructive interference, high impedance points and low impedance points are formed, respectively [4].

The Tesla coil is a circuit known as one of the easiest ways to achieve high voltage, a design also based on a helical resonator. The output voltage value and power consumption depend on the design structure [5]. Quarter wave oscillators are an extremely flexible technology that can be used for such a purpose [6].

Intensive electric field generation based on high-voltage, ultrafast switching is an easy way to obtain a broad spectrum of high-power microwave (HPM) impulses with many potential applications, including medical treatment, product processing, ultra-wideband (UWB) radar, and electric device testing under an electromagnetic field. Other highvoltage applications may include inert gas welding, electrotherapy, and vacuum leak checking [7-11].

While the conventional X-ray generators are using components that are not commonly available, such as turbomolecular pump, X-ray tubes, filament power supply, or constant high-voltage power supplies, the currently implemented helical resonator design will result in a smaller size and possibly higher efficiency as the operating frequency reaches values close to the X-ray domain. Thus, such helical resonators could be used to generate intense electric fields while also generating $X$-ray radiation. Based on the helical antenna mathematics, multiple quarter-wave oscillators' mathematics were determined, having various operating frequencies and covered in the following sections.

\section{Materials and Methods}

The previously presented methods could generate the required oscillations to operate the coil as a helical resonator. Usually, an air-core transformer is used to transfer the excitation signal. It acts as an ideal inductor, but its coupling coefficient varies as the operating frequency changes. A low coupling coefficient is required for the resonator that follows this study to create a medium in which voltage magnification due to standing wave is achieved.

A typical high-voltage air-core transformer is presented below in Figure 1. The secondary will have a low coupling coefficient as a quarter-wave oscillator. At the same time, a low coupling coefficient makes possible a medium in which constructive interference contributes to the output voltage, forming a standing wave from the incident wave and the reflected wave summation.

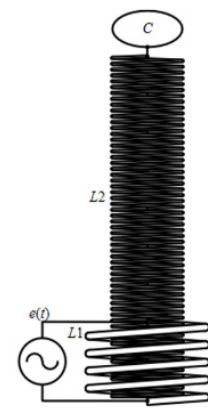

(a)

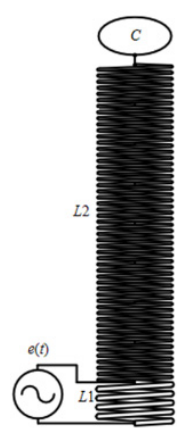

(b)

Figure 1. Helical resonator air-core transformer, Reprinted from Janis Voitkans and Arnis Voitkans (2015) [12]. Possibility for primary side natural frequency adjustment: (a) L1 has a low inductance value due to winding pitch and area; (b) L1 has a higher inductance value compared to the previous case. 
For a low-loss, non-radiating, quarter-wave line with an open circuit at the load end, the voltage magnification ratio at resonance between the top and bottom of the resonator is calculated using Equation (1), where $\alpha$ is the line damping factor, and $h$ represents the length [13]:

$$
\frac{V_{\text {top_load }}}{V_{\text {initial }}}=-j \frac{1}{\alpha h}
$$

In this equation, the left operand represents the voltage magnification ratio.

Calculating the phase shift resulting from the right operand transfer function further correlates the model with the mathematical part and the experimental part. These three sections are covered in the following chapters.

Having the secondary winding operating in the radio waves domain, each time the wave enters a different medium, part of the radiation will reflect into the conductor. The reflection factor can be calculated based on several constraints [14].

As the signal propagates at the speed of light through a conductor, Equation (2) can be used to match the secondary resonant frequency to one quarter wavelength multiple, considering an ideal transmission line.

$$
\frac{4}{(2 N-1)} L=\frac{v_{p}}{f_{s w}}
$$

Equation (2) is another form of Equation (3), presented below:

$$
\lambda=\frac{v_{p}}{f_{s w}}
$$

providing the relation between several variables described below.

These variables represent:

1. $N$-number of maximum points due to standing wave;

2. L-secondary winding wire length;

3. $v_{p}$-propagation velocity, usually considered as the speed of light;

4. $f_{s w}$-transmission line switching frequency;

5. $\lambda$-wavelength of propagated signal.

An illustration for these equations is presented below in Figure 2, where maximum and minimum points due to interference are identified. In any marked maximum points, a reflection of the illustrated incident wave will overlay the reflected wave onto the incident wave. Through repeated summation, the standing wave is formed.

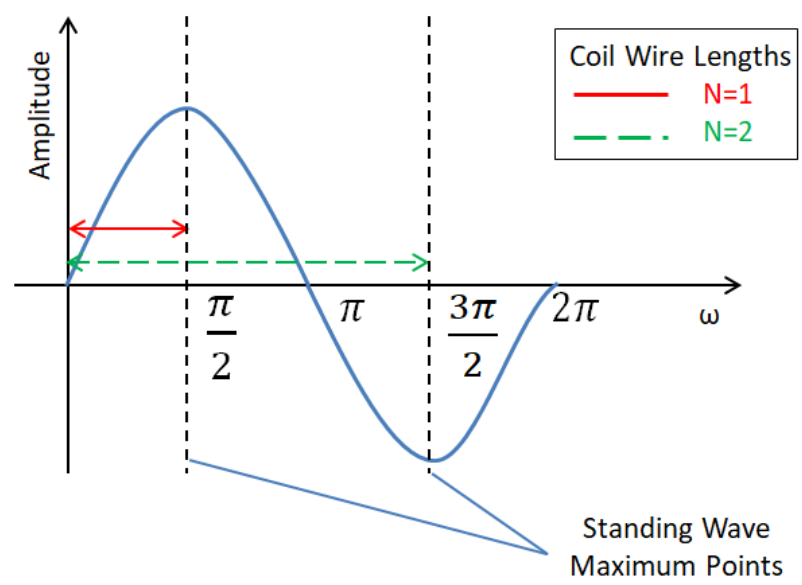

Figure 2. Illustration of secondary wire length versus the propagated wave for one-quarter and three-quarters wavelength resonators. 
A typical high-voltage air-core transformer is presented below in Figure 1. Having a low coupling coefficient, the secondary will act as a quarter-wave oscillator. At the same time, a low coupling coefficient makes possible a medium in which constructive interference contributes to the output voltage, forming a standing wave from the incident wave and the reflected wave summation.

Based on the equation presented above, maximum and minimum electric field values can be identified from Figure 3 .

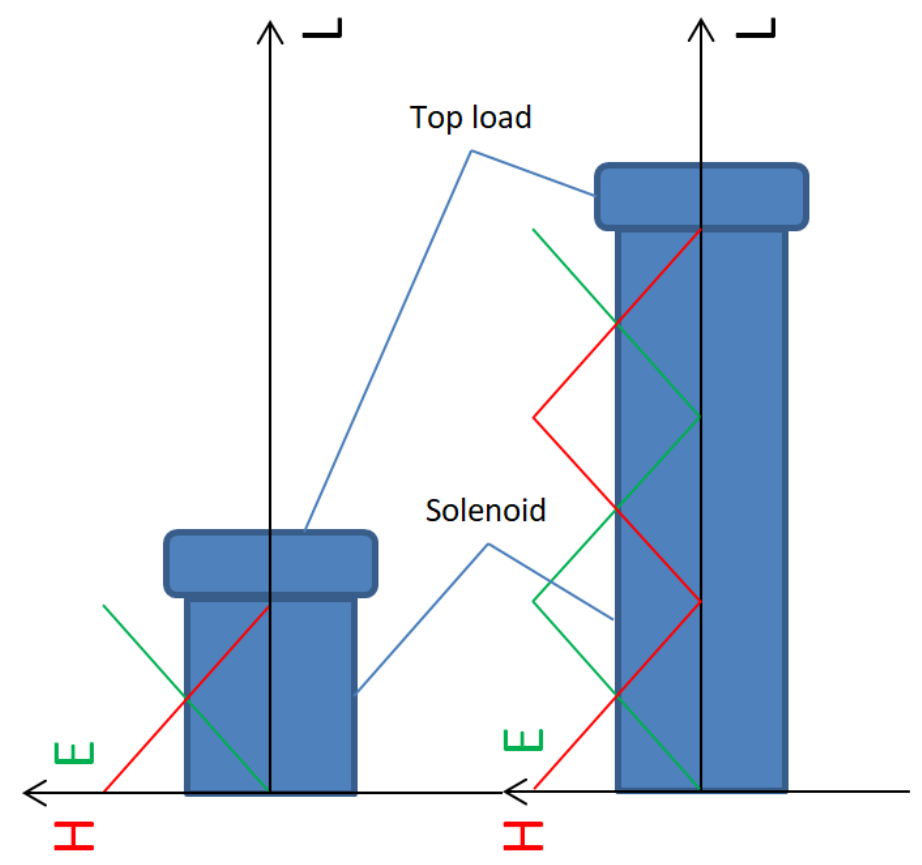

Figure 3. Field distribution in quarter-wave and three-quarter wave topologies (ideal transmission line).

In Equation (2), one can choose different values for the number of maximum points. However, as shown in Figure 3 on the left side for the quarter-wave topology, the electric field vector is concentrated in one maximum point compared to the two maximum points for three-quarters of the wavelength. In the previous equation, the winding length is a function of wavelength.

Having a higher value for $N$, various coupling factors for certain transformer cores can be achieved by choosing a higher resonance frequency. Moreover, choosing very high values for $N$, the number of maximum points will increase the operating frequency indefinitely. In contrast, the typical primary side circuit will require very low values at the X-ray frequency domain.

As the operating frequency increases, the coupling factor inside an air-core transformer becomes better and allows for smaller resonators. However, high-voltage and high-frequency sine wave oscillators represent another challenge, the reason for which the typical spark gap is used to achieve the required oscillations.

The parasitic capacity for an ideal solenoid inductor can be approximated using Equation (4). However, the model used in determining the capacitance is based on $[15,16]$ if we consider obtaining the parameter using the inductance of an insulated copper wire as well as winding pitch, as shown in Equations (5), (7), (9) and (10). Higher capacitance is obtained for more maximum points since this parameter is specific to both the inductor, as shown below, and the propagation mode.

$$
C_{\text {parasitic }}=\frac{2 \pi D}{\operatorname{arccosh}\left(\frac{p}{d}\right)},
$$


where the variables have the following meanings:

6. $C_{\text {parasitic }}$ - parasitic capacitance;

7. $D$-winding diameter;

8. $p$-winding pitch;

9. $d$-wire diameter.

The approach used in this study is shown below in Equation (5).

$$
L=\frac{\mu_{0} \cdot N^{2} \cdot A}{l}
$$

where $(l)$ represents the length. However, according to [16], a more accurate approach can be made to find the secondary side inductance of the helical resonator, as shown in Equation (6).

$$
L=\frac{\mu_{0} \cdot N \cdot A}{\tau}
$$

where the variables mean:

10. L-secondary side inductance;

11. $\mu_{0}$-vacuum magnetic permittivity;

12. $N$-number of windings on the secondary side;

13. A-cross-sectional area of winding;

14. $\tau$-winding pitch (distance between two distinct windings).

The natural frequency is determined only by Equation (2), considering the total wire length approximately as the number of windings multiplied by cross-sectional area circle length, while the parasitic capacitance results from Equation (6). The simulation model uses the calculated parasitic capacitance resulting from the resonant frequency and inductance substitutions.

$$
f_{\text {resonance }}=\frac{1}{2 \pi \sqrt{L C}}
$$

where the variables mean:

15. $f_{\text {resonance }}$-natural frequency;

16. L-secondary side inductance;

17. C-distributed parasitic capacitance.

By choosing a certain capacitor value to match the two frequencies on the primary side, further tuning can usually be made by changing the inductance. The inductance, which is proportional to the vacuum magnetic permittivity $\left(\mu_{0}\right)$, can be tuned using either the number of spires $(N)$ or length $(l)$ with a fixed cross-section area $(A)$.

Ideally, the primary winding number of spires from the air-core transformer should be small to obtain a high-voltage gain using the air-core transformer. Higher frequency involves better coupling coefficients for air-core transformers. Further compensation in model design can be made by calculating the reflected impedance of the secondary side, as shown below in Figure 4.

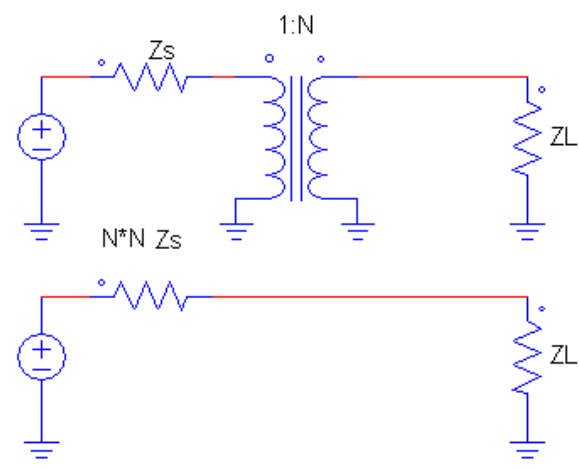

Figure 4. Calculating the reflected impedance. 
As shown above, the reflected impedance was determined as a function of the transformer ratio. The reflected impedance depends on the current supplied by the transformer, which is described using Equation (8).

$$
\frac{N_{p}}{N_{s}}=\frac{V_{p}}{V_{s}}=\frac{I_{s}}{I_{p}}
$$

\section{Results}

This section calculated parameters for the actual experimental design for both primary and secondary sides. In addition, a comparison between the experimental results and simulated SPICE (Simulation Program with Integrated Circuit Emphasis) model is presented.

\subsection{Circuit Components}

\subsubsection{Secondary Side}

For calculating all circuit components, the sizing of the components is initiated using several input variables on the secondary side, as shown below in Table 1.

Table 1. Secondary side parameters.

\begin{tabular}{ccc}
\hline Secondary Side Variable & Meaning & Calculated Value \\
\hline$N$ & Winding number & $500^{1}$ \\
$\mu_{0}$ & Vacuum magnetic permittivity & $4 \pi \times 10^{-7} \mathrm{Hm}^{-11}$ \\
$r$ & Sectional area radius & $10.5 \mathrm{~mm}^{1}$ \\
$\tau$ & Winding distancing & $0.45 \mathrm{~mm}^{1,2}$ \\
$A$ & Sectional area & $3.4 \mathrm{~cm}^{2}$ \\
$L$ & Secondary winding wire length & $35.3 \mathrm{~m}^{3}$ \\
$f_{0}$ & Natural frequency & $2.1 \mathrm{MHz}^{4}$ \\
$L$ & Secondary side inductance & $0.6 \mathrm{mH}^{5}$ \\
$C$ & Distributed parasitic capacitance & $9.4 \mathrm{pF}^{6}$ \\
\hline
\end{tabular}

${ }^{1}$ Designing started with these variables. ${ }^{2}$ This value corresponds to the AWG25 wire size. ${ }^{3}$ From Equation (9) ${ }^{4}$ From Equation (2), replacing the transmission line length and propagation velocity. ${ }^{5}$ From Equation (5). ${ }^{6}$ From Equation (10), resulting in a capacitance specific to the propagation mode and not specific to the solenoid.

Equation (9) was used to find the total wire length of the secondary side.

$$
L=k \cdot N \cdot 2 \pi r
$$

having $L$ as the length and $N$ representing the number of windings. Variable $k$ was used for further adjusting the correctness of Equation (9), and its value is 1.07.

As shown below, the distributed capacitance results from Equation (10).

$$
C=\frac{1}{4 \pi^{2} f_{0}^{2} L}
$$

where $L$ represents the secondary side inductance and $f_{0}$ the natural frequency.

\subsubsection{Primary Side}

The primary side parameters are presented in Table 2, considering the alreadycalculated secondary side parameters.

In this case, the sizing starts with the high-voltage capacitor. By changing the air core inductor parameters, the impedance was adapted to match the one from the secondary side. The high-voltage transformer must be designed to sustain a continuous short-circuit in its primary winding. 
Table 2. Primary side parameters.

\begin{tabular}{ccc}
\hline Primary Side Variable & Meaning & Calculated Value \\
\hline$N$ & Winding number of the primary side & 13 \\
$\mu_{0}$ & Vacuum magnetic permittivity & $4 \pi \times 10^{-7} \mathrm{Hm}^{-1}$ \\
$r$ & Sectional area radius & $11 \mathrm{~mm}$ \\
$\tau$ & Winding distancing & $1 \mathrm{~mm}$ \\
$A$ & Sectional area & $3.8 \mathrm{~cm}^{2}$ \\
$f_{0}$ & Natural frequency & $1.99 \mathrm{MHz}^{1}$ \\
$L$ & Primary side inductance & $4.02 \mu \mathrm{H}^{1}$ \\
$C$ & Resulting high-voltage capacitance & $1.6 \mathrm{nF}^{1}$ \\
$D_{1}$ & Air spark gap length & $2.5 \mathrm{~mm}^{2}$ \\
\hline
\end{tabular}

${ }_{1}$ A greater capacitor value was used to compensate for a small difference in primary side inductance between the calculated and implemented values, resulting in a natural frequency closer to $2.1 \mathrm{MHz} .{ }^{2}$ This value must be less than the length of an air gap having its breakdown voltage corresponding to the peak voltage of the high-voltage transformer.

\subsection{Simulation Model}

The main experimental circuit consists of an integrated MOSFET (Metal Oxide Semiconductor Field Effect Transistor) driver that switches the transistor on and off. The integrated circuit used in this study for driving the MOSFET transistor is IRS2153DPBF, configured at $75 \mathrm{kHz}$. A flyback transformer generated high voltage, which charges the LC circuit up to the spark gap-breaking voltage. Due to leakage inductances, the Schottky diode prevents high-voltage spikes at the MOSFET drain.

The schematic based on which the experimental prototype was built is presented below in Figure 5, and this circuit is also considered a simulation model. The actual value for a high-voltage capacitor is $1.6 \mathrm{nF}$, having used $2 \mathrm{nF}$ as the initial value followed by impedance tuning.

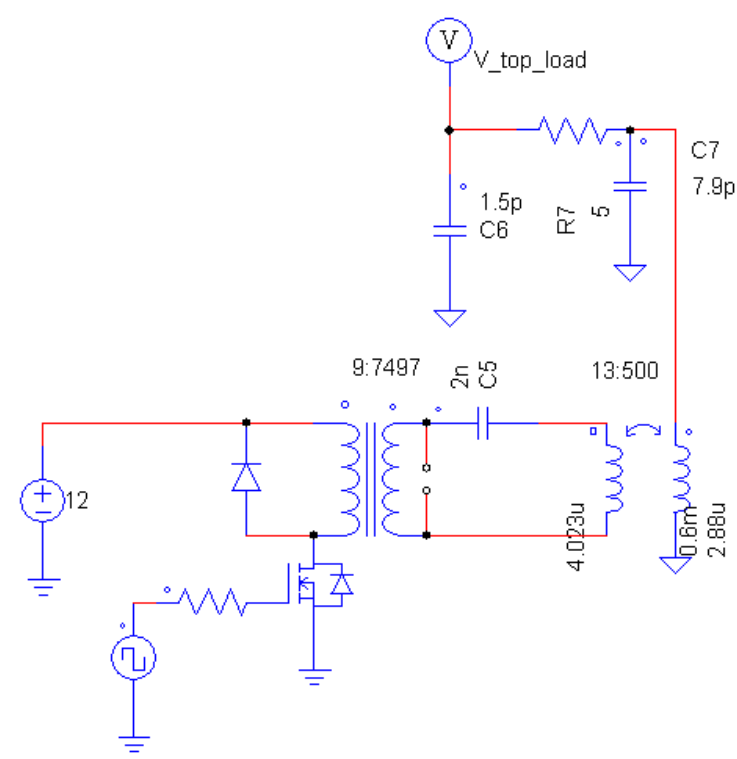

Figure 5. SPICE simulation model, used as a reference in finding the natural frequency of the experimental design.

The oscillating circuits will be separated as the spark gap triggers due to a short circuit occurring at the high-voltage transformer secondary winding. Both LC circuits will enter a state of damped oscillations. For the circuit presented in Figure 4, the time for which the two circuits are separated depends on the gas used for ionization at the spark gap.

By correlating Equation (1) with Figure 5, the resulting phase shift between the incident and top load wave corresponds to the phase shift obtained at resonance for an LC series circuit, which is used as a prototype to describe the natural frequency of the helical resonator. 
Its frequency response is shown below in Figure 6, compared to the experimentally and mathematically confirmed natural frequency.

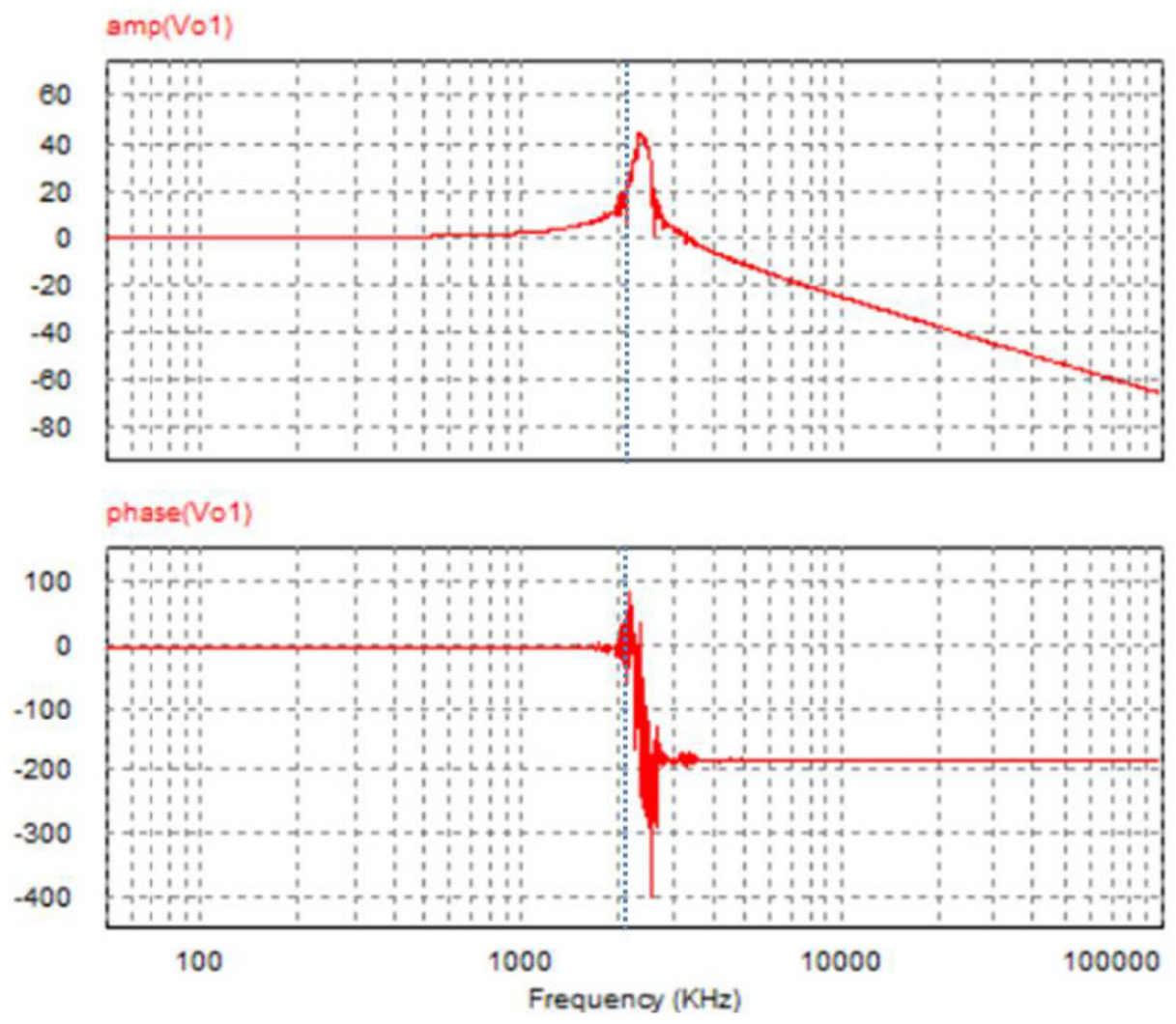

Figure 6. Comparison between natural frequency determined by model (red characteristic) and the mathematically and experimentally confirmed natural frequency (blue dashed line).

The predicted helical resonator natural frequency is $2.4 \mathrm{MHz}$ using the SPICE simulation mathematics, close to the previously obtained result of $2.1 \mathrm{MHz}$ using mathematics and manual calculation described using the previous equations. The difference occurs due to modeling the distributed parasitic capacitance as a series capacitor. The actual natural frequency is determined by transmission line length, having the physical prototype built using $2.1 \mathrm{MHz}$ as natural frequency. The model predicts the natural frequency with a relative error close to $15 \%$ for this design.

The secondary behaves as a transmission line similarly to the previously presented frequency response because the excitation occurs between the secondary top load and its ground terminal, as shown in Figure 7A,B. In this case, the standing wave constructive interference represents an approximate Bode chart gain characteristic.

The previous figure shows that multiple topologies can achieve the standing wave voltage magnification. The reflection coefficient would have a higher value for Figure 7C than Figure 7A or 7B because the air ionizes at both top loads' ends.

\subsection{Physical Prototype}

The simulation model uses a different approach instead of the mathematical operations presented above, used to obtain standing waves due to transmission line reflections. The phenomenon used in the model is a cascade LC series resonance, having the experimental setup built based on several approaches from Table 3. 


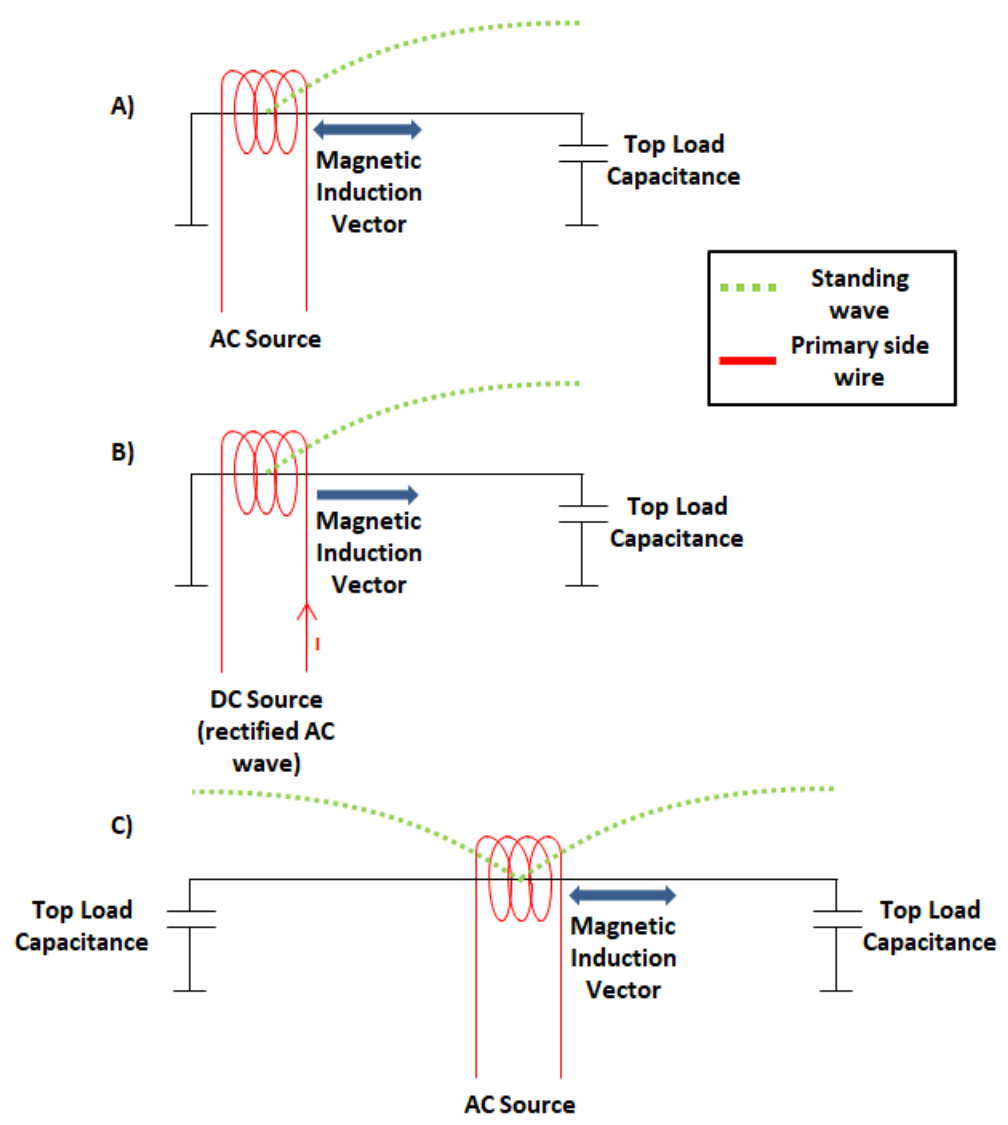

Figure 7. Helical resonator propagation modes at one-quarter of wavelength. An air-core transformer is formed between the primary side winding (red line) and secondary side wire (black line). In all cases, the current must propagate through the secondary side as sinking and/or sourcing the helical resonator top load capacitors, depending on circuit design. (A) The primary side current direction is arbitrary; the standing wave has one maximum point. (B) The secondary side current direction is found using Lenz's Law and Fleming's Right-Hand Rule, assuming the illustrated primary side current; similarly, the propagation leads to one single maximum point. (C) The primary side current direction is arbitrary; wave propagation through the secondary side leads to two maximum points.

Table 3. Data used in building the experimental prototype: mathematical approach based on the previous equations (Method 1, manual calculation), cascade LC resonance model (Method 2, mathematics by model), or experimental (Method 3, trial and error).

\begin{tabular}{cc}
\hline Experimental Prototype Part & Method Used \\
\hline Step-up voltage circuit & Method 3 \\
Primary side parameters frequency matching to the & Method 2 \\
secondary side & Method $1^{1}$ \\
Secondary side natural frequency & Method $3^{1,2}$ \\
\hline Secondary side top-load capacitance &
\end{tabular}

${ }_{1}$ Influenced by secondary side top-load capacitance. ${ }^{2}$ Experimentally, it has been found that the top-load capacitance influences the secondary side natural frequency. Three different top loads were tested.

The helical oscillator circuit is shown below in Figure 8. The most important components were highlighted.

This design uses a 50W flyback transformer to charge the high-voltage capacitors. The voltage gain will vary for higher power resonators-the top-load capacitance should be higher, in this case, to obtain the maximum voltage due to standing wave.

The primary side resonant frequency was calculated using Equation (7). 


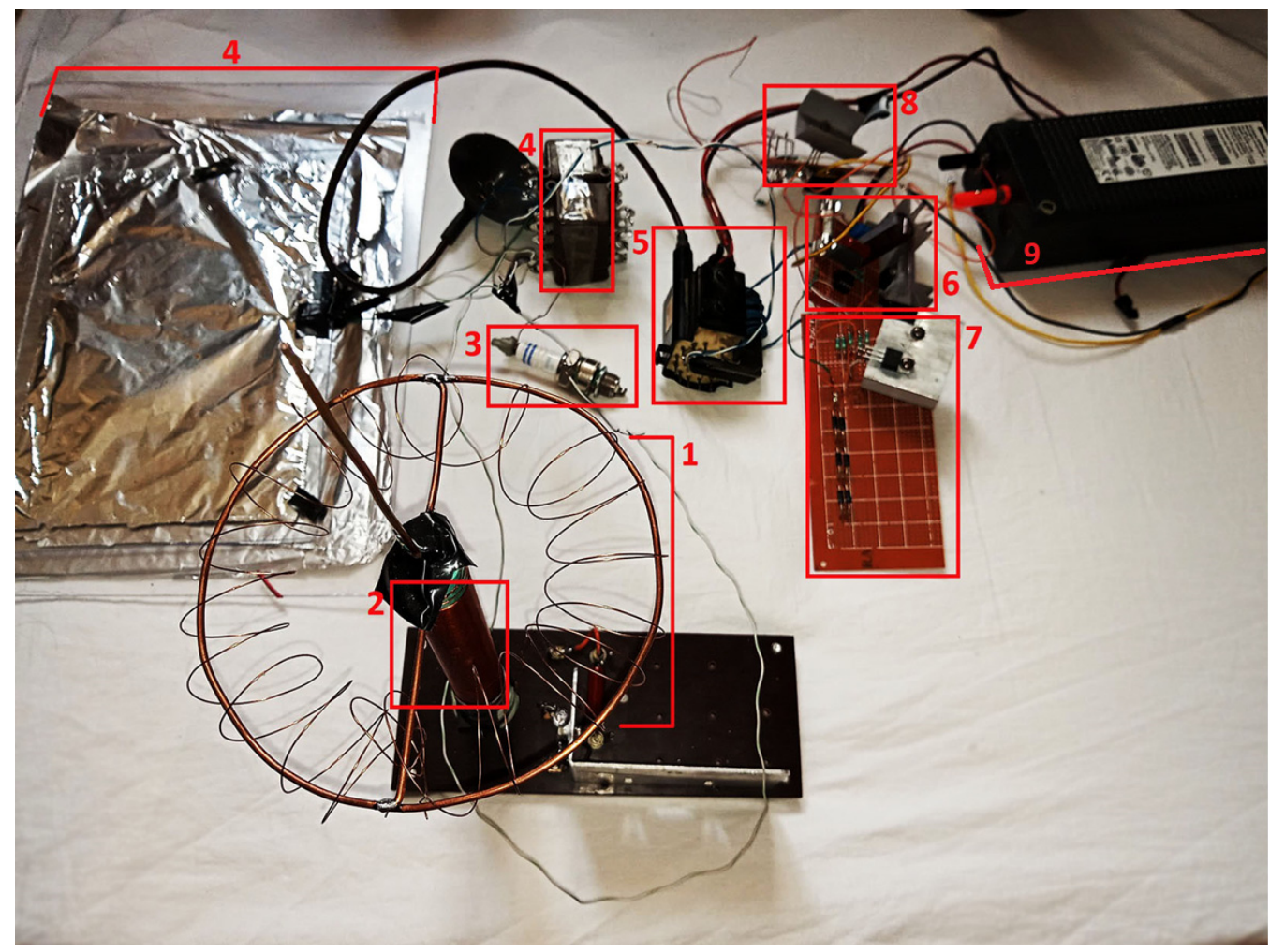

Figure 8. Physical prototype components (detailed explanation is given below).

All values correspond to the variables from Tables 1 and 2. From Figure 8, the following components can be distinguished:

1. Top load torus. This part represents the top load capacitance

2. Helical resonator secondary winding. The primary winding is overlaid at the bottom of secondary onto this part and insulated. An air-core transformer is implemented here, corresponding to the coupled inductors from Figure 5;

3. Spark gap (gasoline engine spark plug). As represented in Figure 5. Distancing is $2.5 \mathrm{~mm}$;

4. High-voltage capacitors. The bank from the left has a total capacitance of $0.6 \mathrm{nF}$, while the one from the right has $1 \mathrm{nF}$. These components are connected in parallel;

5. Flyback transformer. This component is used to supply power to the high voltage circuit, namely components connected to the primary side of the air-core transformer;

6. MOSFET control circuit. This part uses the IRS2153DPBF integrated circuit, keeping an oscillating magnetic flux through the primary side of the high voltage transformer;

7. MOSFET mounted on heatsink and overvoltage protection circuit;

8. Optional modulation circuit. An "AND" logic operation is performed on the MOSFET gate, allowing this resonator to control the output power (number of spark gap breaks per second) in an open loop. The control signal is a PWM (pulse width modulation) signal;

9. DC power supply. Its output voltage is close to 12 volts. This supply is used to emulate a solar panel or geothermal-probe-regulated output voltage. The average supplied current is around 4 amperes for this setup.

The design presented in Figure 8 corresponds to the circuit presented in Figures 5 and 7A. The frequency response was run for the corresponding LC series circuit having a winding resistance of $5 \mathrm{ohms}$ to test the LC filter functionality and impedance matching. Figure 6 shows that the natural frequency is around $2.4 \mathrm{MHz}$ without considering the other constraints.

The actual natural frequency of both the primary and secondary side is close to $2.1 \mathrm{MHz}$ due to a difference in the distributed parasitic capacitance model from the simulation. 


\section{Discussion}

Different top loads were used, and different results were obtained regarding the voltage gain, as shown in Table 4 . The terminal capacitance influences both the voltage gain and the natural frequency of the secondary side.

Table 4. Variation in top-load capacitance.

\begin{tabular}{cc}
\hline Top Load & Outcome \\
\hline No top load & $\begin{array}{c}\text { Standing wave not forming, no electric field present } \\
\text { at top load }\end{array}$ \\
$\begin{array}{c}\text { Top load from Figure } 8 \text { (approximately } 0.5 \text { to } 1.5 \mathrm{pF} \text { ) } \\
\text { Approximately } 2 \text { to } 3 \mathrm{pF} \text { top load }\end{array}$ & $\begin{array}{c}\text { The eltic field is present at the top load }{ }^{1} \\
\text { Stand wave not forming, no electric field present } \\
\text { at top load }\end{array}$ \\
\hline
\end{tabular}

1 This parameter corresponds to component C6 from Figure 5.

Due to several tolerances in the distributed parasitic capacitance, it has been found experimentally that the actual resonant frequency represents approximately $85 \%$ of its calculated value from the Bode chart using the model.

In Figure 9, two results are presented that show an electric field close to the top load. Due to the low power of this design and relatively inefficient step-up high-voltage transformer derived from a flyback configuration, the plasma ionization is not visible at the top of the solenoid without an external object through which the discharge occurs.
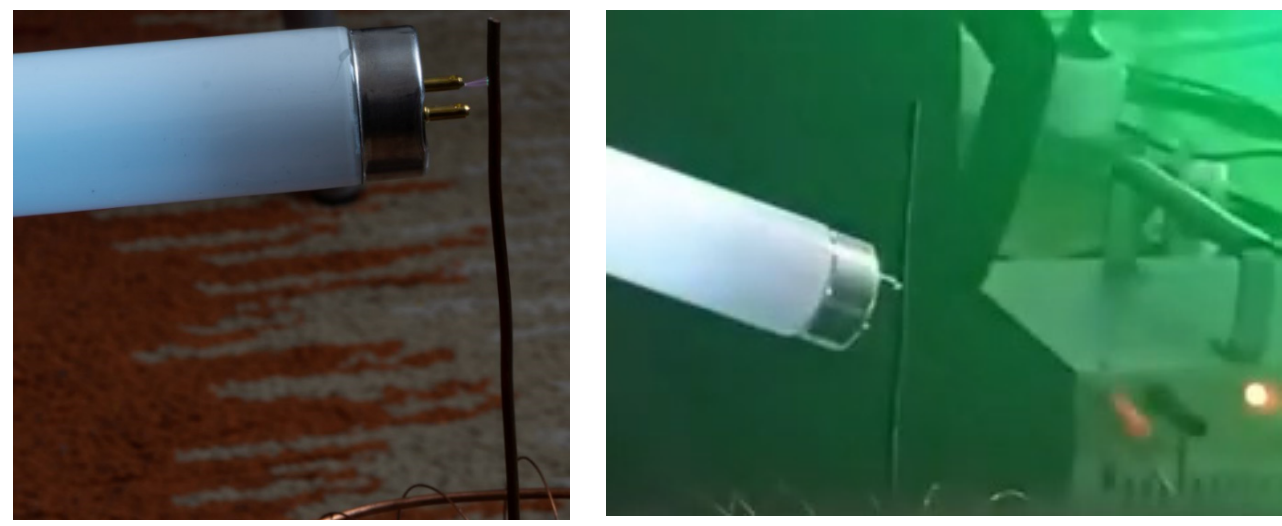

Figure 9. Electric field presence at the top of helical resonator: (a) Discharge without backlight; (b) Discharge shown using green backlight.

Moreover, the study shows that multiple propagation modes result in different selfcapacitance values for the secondary side. The actual parasitic capacitance is specific to the solenoid, not the propagation mode. Further influence of this capacity on the relative error in natural frequency mathematics remains an open point.

Last but not least, Figure 10 shows a possible improvement for this design, which was already designed for operating at low voltage values, such as the outputs of geothermal probes or solar panels.

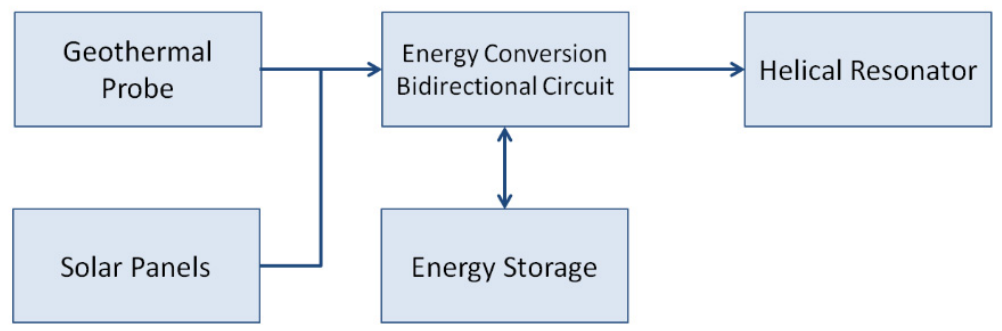

Figure 10. Further circuit improvement with sustainable energy. 


\section{Conclusions}

First and foremost, as shown by equations, standing wave constructive interference occurs inside any conductor when given an ideal power supply. In addition, the secondaryside frequency of a helical resonator was found based on the effect of the same phenomenon, representing a constraint in PCB design, namely transmission line reflections. Moreover, using conventional SPICE simulation software, a model was built to predict the natural frequency of such resonators using a different approach, the LC series resonance, considering the insulated wire capacitance and inductance. This method used by the model was initially thought to be the reason for voltage magnification, having the model using the parasitic capacitance as resulting from Equation (10) for one maximum point. Other scenarios for implementing such models would further investigate the differences that appear for more than one maximum point.

The study shows that standing wave does occur only due to transmission line reflections, meaning that a further improvement to this design would be an even smaller size and higher operating frequency. Furthermore, given an ideal power supply capability, an $X$-ray generator would be indefinitely small due to a very short transmission line in which repeated wave reflections and summations occur, forming stationary waveforms. Moreover, the study shows that such helical oscillators could also be used as X-ray generators.

Within this application, we also aimed to implement a low-power helical resonator that uses the stationary wave summation to concentrate the pulsating electric field in a single point as resulting from mathematics, thus achieving a high voltage gain. An innovative feature of this design is that it fully uses a flyback-derived step-up transformer starting at low voltage to achieve voltage magnification, possibly further improving this design for renewable energy. Thus, in the case of improving this design or using another topology (greater " $\mathrm{N}$ " values) for X-ray or ozone generation, better results can be obtained in certain conditions. However, due to several approximations when using a model, the actual natural frequency will differ from the calculated resonant frequency, as resulted from the model.

While implementing the helical resonator, we aimed to use low-tolerance components and a high quality-to-price ratio. The study of this topic involved several stages. First, for a given number of windings, the coil's natural frequency was calculated as a function of inductance and self-capacitance in inductors, followed by a comparison to mathematical and experimental results. The high-voltage circuit was designed to match the two oscillating frequencies.

In conclusion, following the specialized studies for each block component presented in this work and the simulations of electrical circuits, respectively building the prototype for obtaining the plasma ionization, the practical and functional implementation of the system was successful.

Author Contributions: Conceptualization, methodology, validation, supervision, project administration, funding acquisition, review and editing was done by L.A.S.; Conceptualization, methodology, validation, software, investigation, data curation, writing - original draft preparation, visualization was done by M.A.C. All authors have read and agreed to the published version of the manuscript.

Funding: This research was funded by the Romanian Ministry of Education and Research, grant number PN-III-P2-2.1-PED-2019-2601, “REGRENPOS”.

Conflicts of Interest: The authors declare no conflict of interest.

\section{References}

1. Shams, H.; Gabal, H.A.; Soliman, M.; Ebrahim, S.; Agamy, S. Development of CdS/CdTe Diode for X-Ray Sensor. WSEAS Trans. Circuits Syst. 2020, 19, 268-276. [CrossRef]

2. Rhee, H.S.; Kim, D.H.; Nawaz, S.; Yoon, S.J. Experimental investigation of high efficient inductively coupled plasma by splitting external antenna coil. In Proceedings of the 2017 IEEE International Conference on Plasma Science (ICOPS), Atlantic City, NJ USA, 21-25 May 2017; p. 1. [CrossRef] 
3. Orellana, L.; Ardila-Rey, J.; Avaria, G.; Diaz, M.A.; Pavez, C.; Schurch, R.; Soto, L. On the Relationship between the Electromagnetic Burst and Inductive Sensor Measurement of a Pulsed Plasma Accelerator. IEEE Access 2019, 7, 133043-133057. [CrossRef]

4. Barsoum, N.; Stanley, G.I. Design of High Voltage Low Power Supply Device. Univers. J. Electr. Electron. Eng. 2015, 3, 6-12. [CrossRef]

5. Krbal, M.; Siuda, P. Design and construction solution of laboratory Tesla coil. In Proceedings of the 2015 16th International Scientific Conference on Electric Power Engineering (EPE), Kouty nad Desnou, Czech Republic, 20-22 May 2015; pp. 311-314. [CrossRef]

6. Armanious, M.; Tyo, J.S.; Skipper, M.C.; Abdalla, M.D.; Prather, W.D.; Lawrance, J.E. Interaction Between Geometric Parameters and Output Waveforms in High-Power Quarter-Wave Oscillators. IEEE Trans. Plasma Sci. 2010, 38, 1124-1131. [CrossRef]

7. Schoenbach, K.H.; Joshi, R.P.; Kolb, J.F.; Chen, N.; Stacey, M.; Blackmore, P.F.; Buescher, E.S.; Beebe, S.J. Ultrashort electrical pulses open a new gateway into biological cells. Proc. IEEE 2004, 92, 1122-1137. [CrossRef]

8. Kumar, P.; Baum, C.E.; Altunc, S.; Buchenauer, J.; Xiao, S.; Christodoulou, C.G.; Schamiloglu, E.; Schoenbach, K.H. A hyperband antenna to launch and focus fast high-voltage pulses onto biological targets. IEEE Trans. Microw. Theory Tech. 2011, 59, 1090-1101. [CrossRef]

9. Xiao, S.; Guo, S.; Nesin, V.; Heller, R.; Schoenbach, K.H. Sub-nanosecond electric pulses cause membrane permeabilization and cell death. IEEE Trans. Biomed. Eng. 2011, 58, 1239-1245. [CrossRef] [PubMed]

10. Kohler, S.; O'Connor, R.P.; Vu, T.D.T.; Leveque, P.; Arnaud-Cormos, D. Experimentalmicrodosimetry techniques for biological cells exposed to nanosecond pulsed electric fields using microfluorimetry. IEEE Trans. Microw. Theory Tech. 2013, 61, 2015-2022. [CrossRef]

11. Beebe, S.J.; Fox, P.M.; Rec, L.J.; Somers, K.; Stark, R.H.; Schoenbach, K.H. Nanosecond pulsed electric field (nsPEF)effects on cells and tissues: Apoptosis induction and tumor growth inhibition. IEEE Trans. Plasma Sci. 2002, 30, 286-292. [CrossRef]

12. Voitkans, J.; Voitkans, A. Tesla Coil Theoretical Model and it's Experimental Verification. Electr. Control Commun. Eng. 2014, 7, 11-19. [CrossRef]

13. Corum, K.L.; Corum, J.F. RF Coils, Helical Resonators and Voltage Magnification by Coherent Spatial Modes. In Proceedings of the 5th International Conference on Telecommunications in Modern Satellite, Cable and Broadcasting Service, Nis, Yugoslavia, 19-21 September 2001.

14. Ligthart, L.P.; Kozlov, A.I.; Logvin, A.I.; Avtin, I.V. Radio-waves Reflection at Remote Sensing of Underlying Covers. Civ. Aviat. High Technol. 2019, 22, 57-66. [CrossRef]

15. Grandi, G.; Kazimierczuk, M.K.; Massarini, A.; Reggiani, U. Stray capacitance of single-layer solenoid air-core inductors. IEEE Trans. Ind. Appl. 1999, 35, 1162-1168. [CrossRef]

16. Siverns, J.D.; Simkins, L.R.; Weidt, S.; Hensinger, W.K. On the application of radiofrequency voltages to ion traps via helical resonators. Appl. Phys. B 2012, 107, 921-934. [CrossRef] 